\title{
Commission 4. (EPHEMERIDES.)
}

Professor W. S. Eichelberger, President of the Commission, presided. Dr Jackson acted as Secretary.

The President announced that the resolution concerning the use of I950.0 as a standard equinox for cometary and asteroid work had been discussed in Commission 20 which approved the arrangements which have been made by the Directors of the National Ephemerides to change over to the equinox of 1950.

The subject was not discussed further.

The resolution concerning the adoption of fixed dates for elements and ephemerides of comets and minor planets had been discussed in Commission 6 where a definite arrangement had been agreed upon.

The Commission then discussed the two proposals on the terminology of time. After considerable discussion in which it was made clear that the British Nautical Almanac was committed to use the letters G.M.T. for time counted from Greenwich Mean Midnight, the following was adopted:

"In all astronomical work, times given should indicate clearly whether the day used begins at noon or midnight."

Dr Jackson referred to the work which he is doing in collaboration with Dr Knox-Shaw in the reduction of the observations made by Hornsby at Oxford in I774-I803. In order to fix the equator of the instrument a complete ephemeris of the sun, based on Newcomb's tables and computed with modern accuracy, has been made for every day in the years $1775-1784$ and I790-1796. To check the rotation of the earth, and also the equator point, it is desirable to reduce the numerous observations of planets. Mr Bawtree, formerly of the British Nautical Almanac Office has agreed to compute an ephemeris of Venus for I780-I784. Offers of help in further calculations of planetary ephemerides were asked for.

Dr Jackson also mentioned that in the determination of the equator point the minor planet Vesta had certain advantages over the sun and the major planets and he suggested that it would be useful to have tables of this planet computed with Newcomb's values of the masses of Jupiter and Mars.

The members of the Commission present at the Leiden Meeting, MM. Andoyer, Brown, Eichelberger, Herrero, Jackson and Volta, submitted the following:

The terms Greenwich Civil Time (G.C.T.), Weltzeit (W.Z.) and Universal Time (U.T.) denote time measured from Greenwich Mean Midnight, and are not ambiguous. The name Greenwich Mean Time (G.M.T.) for dates before I925 Jan. I, refers to time counted from Greenwich Mean Noon, but as used after that date in British publications it refers to time counted from midnight. Astronomers are advised not to use the letters G.M.T. in any sense for the present.

Some astronomers desire to employ time counted from Greenwich Mean Noon. For that purpose the letters G.M.T. are no longer available. The expression Greenwich Mean Astronomical Time (G.M.A.T.) though long is the most satisfactory for describing time counted from Greenwich Mean Noon. The Julian day is counted from Greenwich Mean Noon.

\section{Commission 5. (BIBLIOGRAPHIE.)}

La Ve Commission s'est réunie à deux reprises différentes, sous la présidence de M. Stroobant, afin de discuter en détail le rapport qui avait été présenté par son secrétaire, ainsi que les résolutions consécutives proposées à ses délibérations (voir pp. 19-39). 
M. Mascart a résumé la genèse de son rapport. A la suite des discussions de Cambridge (I925) il a établi un projet préliminaire qu'il a publié à ses frais dans le Bulletin de l'Observatoire de Lyon (Janvier I927): ce projet fut envoyé à tous les membres de l'Union, avec prière de le retourner annoté, corrigé et complété autant que possible; c'est en tenant compte de la majorité des desiderata exprimés alors que le rapport définitif fut établi, de sorte qu'il semble bien refléter désormais l'opinion générale.

Personne ne demandant la parole pour discuter les questions de principes, plan et méthodes, la Commission passe à l'examen des résolutions présentées.

Les deux premières résolutions sont adoptées sans modifications.

Pour la $3^{\mathrm{e}}$ résolution, $\mathrm{M}$. Turner montre que le résumé est plus avantageux au début de l'article, ce qui conduit à rédiger le paragraphe: soit, surtout, au début.... M. de Vos van Steenwijk fait adopter un $2^{\theta}$ paragraphe pour appeler l'attention dés directeurs de publications sur l'importance de cette résolution.

La $4^{\ominus}$ résolution est adoptée sans modifications.

A propos de la $5^{\ominus}$ résolution, M. Mascart fait remarquer qu'il ne s'agit ici que d'une approbation de principe, mais que cette consécration de ses efforts peut lui être fort précieuse pour trouver des ressources propres à développer sa tentative si l'Union estime qu'elle peut rendre des services. M. Kopff déclare que la Bibliographie rapide faite est très digne d'être encouragée et qu'elle lui rend à lui-même de grands services, déclaration qui est soulignée par des applaudissements: une telle approbation prenait une valeur particulière, et par la haute compétence du Directeur de l'Astronomischer Jahresbericht, et aussi par ce fait que, dans les critiques recueillies sur le premier projet, quelques astronomes paraissaient craindre qu'il y eut quelque double-emploi et la possibilité d'une petite rivalité entre publications différentes. On se rend bien compte désormais que, poursuivant des buts distincts et sans la moindre arrière-pensée de concurrence, plusieurs astronomes se dévouent à une œuvre d'information et d'utilité générale, trop heureux de pouvoir se rencontrer pour discuter des points de détail afin de faire converger leurs efforts vers des objectifs pratiques.

La $6^{\oplus}$ résolution est adoptée sans modification.

La $7^{\ominus}$ résolution, sur la proposition de M. de Vos van Steenwijk, comportera une légère modification de rédaction.

M. Turner fait adopter une résolution relative à la technique de la distribution des subventions de l'Union; combattu par le bureau de l'Union, cette résolution fut retirée devant 1'Assemblée Générale.

La Commission propose trois subventions pour la publication (8) de la Bibliographie du Bulletin de Lyon, de la liste (9) des observatoires, et (Io) des publications.

M. de Vos van Steenwijk fait discuter deux importantes questions qui ne peuvent être résolues sans des accords avec le Congrès International de Linguistique et l'Institut International de Coopération Intellectuelle; la Commission conclut à la rédaction de deux résolutions.

On trouvera ailleurs (p. 300) le texte exact des résolutions adoptées en Assemblée Générale de l'Union.

\section{Commission 6. (TELEGRAMMES.)}

Professor Elis Strömgren, President of the Commission, presided over two meetings, which were attended by Sir Frank Dyson and by Dr Shapley. Mr Felix de Roy acted as Secretary.

The printed Report (p. r8o) was adopted. 